\section{Apprêtement des antigènes présentés par les molécules de classe I du CMH}

Peter van Endert

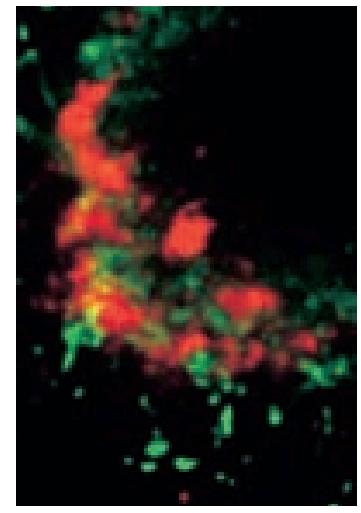

Inserm U580

et Faculté de Médecine, Université Paris 5

Parmi les acteurs de René Descartes, l'immunité adaptative, 161, rue de Sèvres, les lymphocytes T cyto- 75015 Paris, France. toxiques, qui expriment vanendert@necker.fr à leur surface le marqueur CD8, jouent un rôle crucial. Ils reconnaissent et éliminent les cellules qui présentent des fragments peptidiques issus de pathogènes ou de protéines associées à une transformation maligne, et donc considérés comme étrangers par l'organisme. Ce système de reconnaissance repose sur la capacité des molécules de classe I du complexe majeur d'histocompatibilité de fixer ces peptides au sein de la cellule, puis de les présenter à la surface cellulaire [2]. Un système intracellulaire pour la production de ces peptides, et pour leur acheminement vers les molécules CMH de classe I, est donc indispensable pour le bon fonctionnement de nos défenses immunitaires.

Le système d'approvisionnement en peptides doit faire face à différentes contraintes: traiter des peptides de séquences diverses, représentatifs des différents «motifs» de séquence des molécules de classe I qui présentent des variations alléliques; produire des peptides d'une longueur précise, entre 8 et 10 acides aminés, adaptée au sillon des molécules de classe I qui les logera ; les produire rapidement, afin que la réponse immunitaire puisse efficacement limiter le nombre de réplications des pathogènes et leur dissémination; lutter contre des pathogènes ayant des tropismes tis- 
sulaires et subcellulaires divers, et contrer les mécanismes d'évasion de la réponse immunitaire qu'ils sont capables de mettre en place [3].

Le système cellulaire d'apprêtement (processing) des antigènes s'appuie de façon importante sur des molécules qui sont les acteurs «de ménage» du métabolisme cellulaire, mais implique également un certain nombre d'acteurs plus spécialisés, ayant pour fonction exclusive le processing des antigènes. Dans la première catégorie, on trouve les protéases cytosoliques, et notamment le protéasome, qui dégrade les antigènes, ainsi que plusieurs protéines de type chaperon du réticulum endoplasmique $(R \varepsilon)$, ayant pour rôle d'assurer le repliement correct des molécules de classe I, ainsi que leur «chargement ». Dans la deuxième catégorie, on citera un transporteur de peptides situé à la membrane du RE, deux aminopeptidases et une protéine chaperonne de ce compartiment, ainsi que certains composants «optionnels » du protéasome.

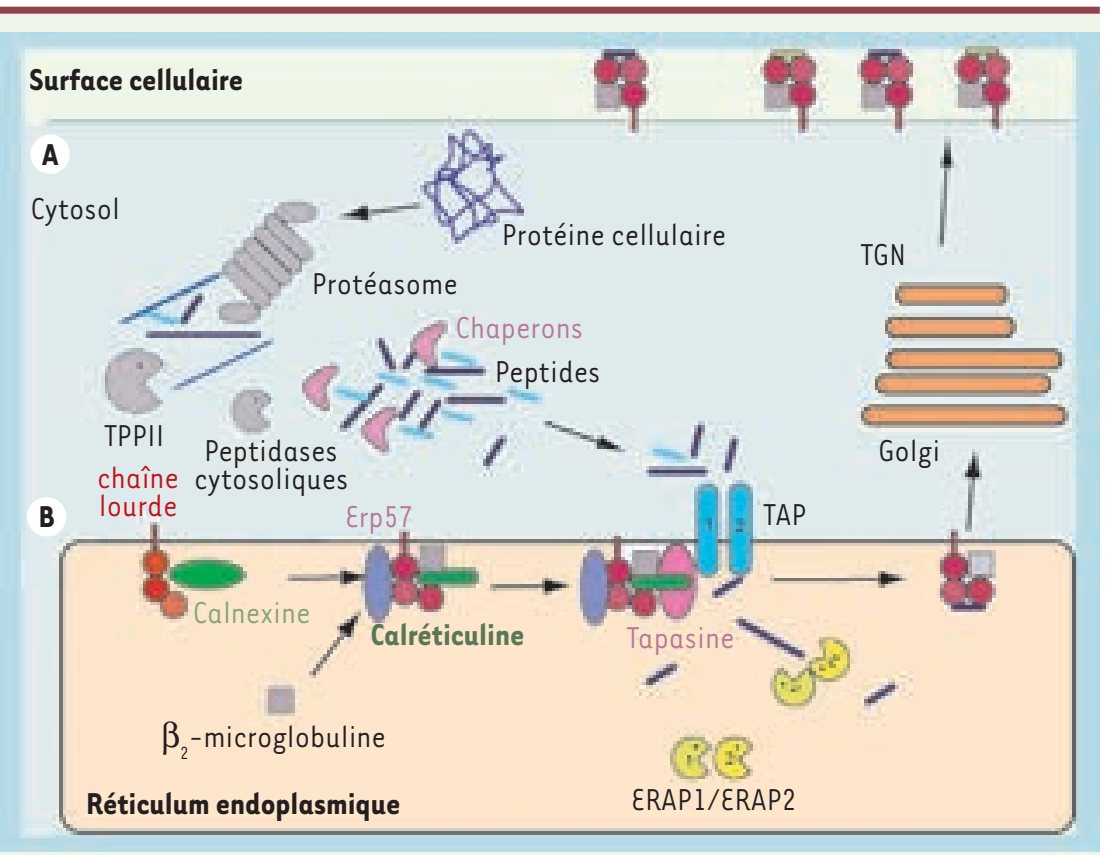

Figure 1. Appêtement des antigènes et complexes de chargement des molécules de classe I du CMH. A. Les protéines synthétisées par la cellule sont dégradées par le protéasome, puis détruites ou élaguées par la tripeptidylpeptidase (TPPII) et les diverses peptidases cytosoliques. Une dernière étape d'élagage par les peptidases ERAP1/2 (endoplasmic reticulum aminopeptidases) peut avoir lieu après le transport des peptides dans le réticulum endoplasmique, grâce aux pompes TAP (transporter associated with antigen processing). B. L'assemblage des molécules de classe I est assisté et contrôlé par plusieurs protéines chaperonnes, dont les lectines calnexine et calréticuline, l'oxydoréductase ERp57 et la chaperonne spécialisée tapasine. Les complexes des chaînes lourdes et légères CMH sont ancrés aux pompes TAP par une interaction non covalente avec la chaperonne tapasine. Les glycanes de la chaîne lourde sont fixés par la lectine calréticuline qui, elle, interagit avec l'oxydoréductase ERp 57, vraisemblablement impliquée dans l'oxydation de la chaîne lourde. ERp57 forme des ponts disulfures avec la tapasine, dont la fonction reste incertaine. Après la liaison d'un peptide de bonne affinité, les molécules de classe I sont libérées et avancent vers la surface cellulaire en passant par le réseau transgolgien (TGN).
Cette revue fait le point sur les différents éléments du processing des antigènes, de la synthèse des protéines sources jusqu'à l'exportation des molécules de classe I chargées en peptides à la surface, en passant par les différentes étapes protéolytiques, le transport du cytosol vers le RE, l'élagage des peptides dans ce dernier et, enfin, le chargement de peptides optimisés au sein des

\section{Protéines sources des peptides}

Les peptides présentés par les molécules de classe I du CMH sont en général issus de la dégradation des protéines endogènes, synthétisées par la cellule présentatrice. Cependant, les cellules dendritiques, qui jouent un rôle central dans l'initiation des réponses cytotoxiques, sont dotées d'une voie de processing supplémentaire, qui leur permet de présenter aussi des antigènes internalisés [4] : il s'agit du phénomène de présentation croisée, ou cross-presentation.

Des deux compartiments subcellulaires dotés d'une capacité protéolytique importante, le lysosome et le cytosol [5], l'approvisionnement des molécules de classe I en peptides s'appuie sur le deuxième. Dans le cytosol, les protéines ayant rempli ou perdu leur fonction sont dégradées par le protéasome, grand complexe protéolytique [6] (Figure 1A). Ce processus ne concerne pas uniquement les protéines cytosoliques, mais aussi un grand nombre de protéines destinées à la voie sécrétoire, puisque les protéines mal repliées ou mal assemblées dans le RE sont expulsées dans le cytosol pour y être dégradées [7]. L'appareil protéolytique du cytosol ne fait pas la différence entre les protéines codées par le génome cellulaire et celles qui sont issues de pathogènes infectant la cellule, les deux étant traitées comme «endogènes ».

En utilisant des peptides issus de la dégradation cytosolique de protéines devenues «obsolètes», le système de processing pour les molécules de classe I se superpose au métabolisme cellulaire des protéines, dont la fonction est d'éliminer les protéines non fonctionnelles et de recycler les acides aminés libérés pour la synthèse de nouvelles protéines. Cependant, et de façon surprenante, le système de proces- 
sing semble directement lié à la synthèse des protéines. Plusieurs groupes ont récemment montré qu'une grande partie des peptides présentés par les molécules $\mathrm{CMH}$ de classe I est issue de la dégradation de protéines d'une très courte durée de vie, dont la majorité semble correspondre à des protéines n'ayant jamais atteint un état fonctionnel (defective ribosomal products, ou DRiP) [8] (Figure 2). Selon ces auteurs, jusqu'à $30 \%$ des produits de la traduction ribosomale seraient défectueux, fournissant ainsi la majorité des peptides présentés par les molécules de classe I [8]. Cette hypothèse implique que le processing des antigènes serait lié de façon plus étroite au taux de traduction des protéines qu'à leur taux de dégradation. L'hypothèse des DRiP, qui d'ailleurs est contestée [9], touche aux phénomènes encore mal compris du processing des antigènes. Les raisons permettant d'expliquer l'apparente courte demi-vie des DRiP, le rôle de ces derniers pour les protéines des différents compartiments cellulaires (cytosol, RE), ainsi que les facteurs déterminant le taux de DRiP d'une protéine donnée doivent encore être précisés. L'effi-

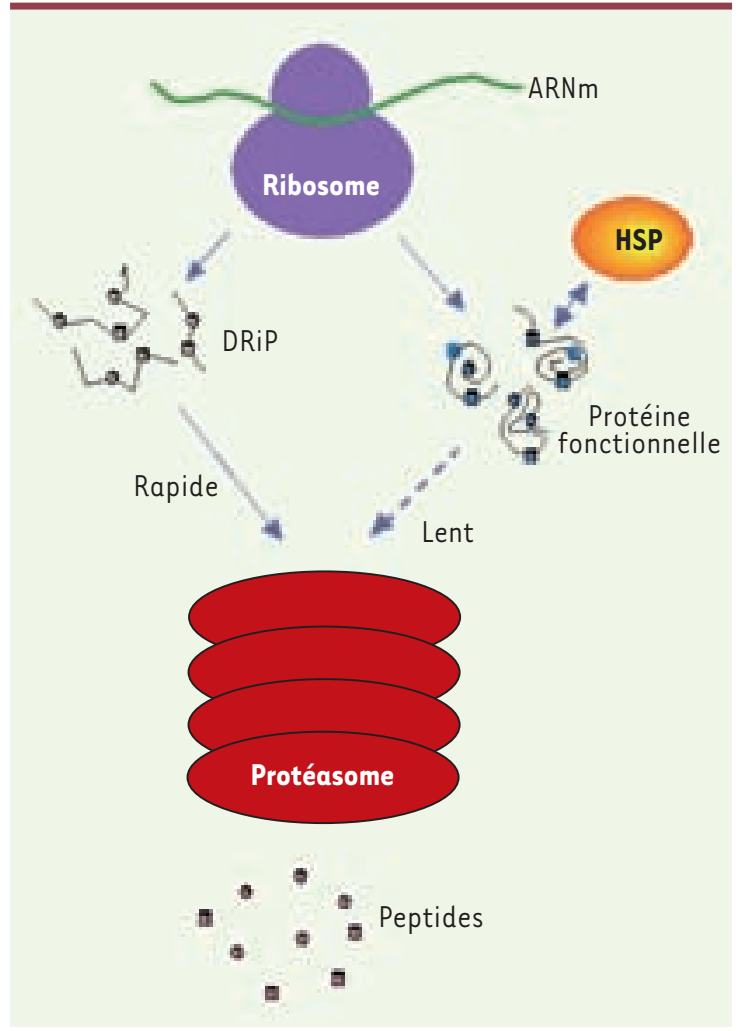

Figure 2. Les DRiP, source importante de peptides présentés par les molécules de classe I du CMH. Une fraction substantielle des protéines traduites par l'appareil ribosomique n'atteint pas leur état fonctionnel (DriP, defective ribosomal products) et subit une dégradation rapide, alors que les protéines ayant une conformation correcte sont dégradées de façon plus lente. Le protéasome dégrade les DRiP comme les protéines en fin de vie. HSP : heat shock protein. cacité surprenante de la présentation de certains peptides reste également mystérieuse. Alors que, en moyenne, plus de 1000 protéines sources doivent être dégradées pour produire un complexe peptide/CMH-I $[10,11]$, certains peptides produits par des mécanismes non conventionnels, et probablement marginaux dans la cellule, tels que la traduction de cadres de lecture alternatifs, anti-sens, ou initiés par des codons autres que la méthionine [12], ou encore l'épissage de peptides [13], provoquent des réponses cellulaires efficaces. Une hypothèse intéressante, mais purement spéculative, est celle de l'existence d'un compartiment, ou domaine, cytosolique dont la fonction principale serait de produire des peptides antigéniques, et où des peptides non conventionnels seraient surreprésentés.

\section{Protéolyse cytosolique des antigènes}

Le protéasome, une protéase abondante composée d'une structure cylindrique formée par 28 protéines organisées en quatre anneaux (complexe 20S), en association avec des «chapeaux » régulant l'accès des substrats aux ouvertures périphériques de la cavité du cylindre (complexe 26S), occupe une place centrale dans le métabolisme des protéines cellulaires [6]. Bien que la grande majorité des études de laboratoire utilisent des complexes $20 \mathrm{~S}$, plus stables in vitro, on considère que, dans une cellule, les complexes $26 \mathrm{~S}$ représentent la forme dominante du protéasome. Ces derniers ne dégradent que les substrats marqués pour la dégradation par une poly-ubiquitinylation, c'est-à-dire l'ajout, par un lien covalent, de châ̂nes d'au moins quatre molécules d'ubiquitine sur des résidus lysine [14]. Les protéines poly-ubiquitinylées sont reconnues par certaines protéines faisant partie des chapeaux 26S, qui contiennent en plus des enzymes capables d'enlever les chaînes poly-ubiquitine, et de dénaturer les substrats protéiques pour permettre leur introduction dans le canal central des complexes 205 donnant accès aux sites catalytiques de la protéase.

Le protéasome est une protéase multicatalytique, dotée de trois spécificités catalytiques qui correspondent à trois sous-unités au sein des anneaux intérieurs des complexes 20S [15]. Ces trois spécificités sont communément qualifiées comme similaires à la trypsine (clivage après les résidus Arg et Lys), à la chymotrypsine (clivage après les résidus Tyr, Phe, Leu et $\|$ e) et à la caspase (clivage après Glu et Asp). Du fait de ces spécificités multiples, le protéasome est capable de dégrader la quasi-totalité des protéines, en produisant des fragments d'une longueur de 4 à 15 résidus. Alors que certains considèrent que moins de $15 \%$ des peptides produits par le protéasome ont une longueur supérieure à celle des peptides présentés par les molécules de classe I du CMH [16], d'autres proposent que les expériences in vitro ne reflètent pas fidèlement l'action du protéasome dans l'environnement cellulaire, et que la longueur de la plupart des peptides produits dépasse en fait 15 résidus [17]. Sous l'influence de l'interféron $\gamma$, la spécificité du protéasome s'adapte de façon plus précise aux besoins de la présentation des antigènes par les molécules $\mathrm{CMH}-\mathrm{I}$. Dans cette situation, le remplacement des trois sous-unités catalytiques du protéasome dénommé « protéasome constitutif » par d'autres sous-unités résulte en la formation de complexes «d'immunoprotéasome » [15]. Ces derniers conservent les spécificités de type trysine et chymotrypsine, mais ne possèdent plus aucune activité de type caspase, inutile à la présen- 
tation d'antigènes par les molécules de classe I, qui ne fixent jamais des peptides ayant un résidu acide en position carboxyterminale.

La très grande majorité (probablement plus de 99,9\%) des peptides produits par le protéasome sont décomposés en acides aminés recyclés pour la synthèse de protéines. Ce constat implique que l'appareil protéolytique du cytosol détruit un grand nombre d'épitopes potentiels, pouvant être présentés par les molécules $\mathrm{CMH}-\mathrm{I}$, une conclusion confirmée par de nombreuses observations expérimentales [15]. Les recherches récentes suggèrent qu'un relais de plusieurs protéases se charge de la destruction des peptides dans le cytosol [18], dont la demi-vie n'est que de quelques secondes [17]. Les peptides d'une longueur supérieure à 15 résidus sont les substrats préférés de la tripeptidylpeptidase, une protéase formant de grands complexes également cylindriques qui pourraient aussi jouer un rôle dans la présentation antigénique [17]. Les peptides d'une longueur de 15 à 9 résidus sont dégradés par la thimet oligopeptidase, et les peptides plus courts par diverses aminopeptidases (leucine aminopeptidase, bleomycine hydrolase et puromycin-sensitive aminopeptidase [18]). De façon remarquable, toutes ces protéases s'attaquent à l'extrémité aminoterminale des peptides. Aucune carboxypeptidase impliquée dans la présentation antigénique en aval du protéasome, ou dans la destruction d'épitopes potentiels dans le cytosol, n'a été décrite à ce jour. On considère que les extrémités carboxyterminales des peptides présentés par les molécules $\mathrm{CMH}-$ I sont quasi exclusivement produits par le protéasome, alors que tout un éventail d'aminopeptidases peut agir sur l'extrémité aminoterminale des épitopes dans le cytosol et dans le RE. L'hypothèse que le cytosol ou le RE pourraient héberger des protéines «navettes», capables de protéger les peptides et de les acheminer vers le lieu d'assemblage avec les molécules $\mathrm{CMH}-\mathrm{I}$, a été à l'origine de plusieurs études. Étant capables de fixer des peptides, les protéines de choc thermique, abondantes autant dans le cytosol que dans le RE, ont été proposées pour cette fonction [19]. Cependant, aucune preuve pour un rôle important de ces protéines dans le processing cellulaire des antigènes n'a été rapportée, ce rôle restant donc hypothétique.

\section{Transport des peptides du cytosol dans le réticulum endoplasmique}

La translocation des peptides antigéniques de leur lieu de production au site où intervient l'assemblage avec les molécules du CMH-I est prise en charge par le transporteur TAP (transporter associated with antigen processing); ce dernier est composé de deux sous-unités homologues et appartient à la grande famille des protéines $A B C$, qui utilisent l'énergie fournie par l'hydrolyse de l'ATP pour transporter des solutés à travers les membranes [20]. La très faible expression de molécules $\mathrm{CMH}-\mathrm{I}$ à la surface de cellules déficientes en TAP témoigne de la nature essentielle de cette pompe, dont l'expression est induite par l'interféron $\gamma$. Les pompes TAP chez les rongeurs, mais aussi chez l'homme, montrent une spécificité pour la longueur et la séquence des peptides qui varie entre les espèces et contribue à la sélection des épitopes immunodominants. Chez toutes les espèces étudiées, TAP semble préférer des peptides d'une longueur de 8 à 16 résidus, ce qui permet le transport de peptides ayant une longueur optimale pour les molécules CMH-I, ainsi que de précurseurs. En revanche, les pompes TAP sont distinctes chez I'homme et chez la souris quant à leurs séquences préférées [21]: alors que le transporteur TAP humain préfère les grands acides aminés hydrophobes (surtout Tyr et Phe) et les résidus basiques (Arg), le transporteur TAP de la souris n'accepte pas ces derniers. Ces préférences sont en parfaite adéquation avec celles des molécules $\mathrm{CMH}-\mathrm{I}$ des deux espèces concernées. Chez l'homme, nos études ont mis en évidence une contribution importante des trois positions aminoterminales pour le transport d'un peptide par TAP, alors qu'une telle analyse n'a pas encore été entreprise pour les pompes chez la souris [22].

\section{Apprêtement et assemblage des peptides dans le réticulum endoplasmique}

Une fois transportés dans le RE, les peptides peuvent subir une ultime étape protéolytique qui permet de produire certains épitopes à partir de peptides précurseurs portant des extensions aminoterminales [23]. Chez I'homme, deux aminopeptidases, ERAPI (endoplasmic reticulum aminopeptidase 1 ) et ERAP2, sont impliquées dans ce processus, alors que la souris n'exprime qu'ERAP1. Les deux enzymes sont induites par l'interféron $\gamma$, peuvent former des complexes hétérodimériques et possèdent des spécificités distinctes, qui leur permettent d'agir de façon complémentaire: ERAP1 préfère les acides aminés hydrophobes, tandis qu'ERAP2 montre une forte préférence pour les acides aminés basiques [24]. Il est intéressant de noter qu'ERAPl digère de façon préférentielle les substrats d'une longueur de 9 à 16 résidus, en parfaite adéquation avec la longueur préférée par les pompes TAP [25].

La formation d'un complexe entre un peptide et une molécule CMH-I implique l'action de quatre protéines chaperons différentes, dont trois appartiennent au système de contrôle de qualité du $R \varepsilon$, qui empêche les protéines dont la conformation n'est pas adéquate de sortir de ce compartiment [26] (Figure 1B). Du fait de leur faible stabilité en l'absence de peptide fixé, les molécules $\mathrm{CMH}-\mathrm{I}$ restent en permanence associées à ces protéines, jusqu'à ce qu'elles acquièrent un peptide de haute affinité. Les chaînes lourdes nouvellement synthétisées sont d'abord associées à la calnexine, une lectine «de ménage» du $R \varepsilon$ qui aide au repliement correct de glycoprotéines. L'association des chaînes lourdes avec la $\beta_{2}$-microglobuline est accompagnée par le remplacement de la calnexine par une deuxième lectine, la calréticuline, ainsi que par l'entrée en jeu de l'ERp57, une oxydoréductase catalysant probablement la formation des ponts disulfures au sein des chaînes lourdes. Les complexes ainsi formés se lient enfin 
directement aux pompes TAP, par l'intermédiaire d'une protéine chaperonne spécialisée, la tapasine, constituant ainsi les «complexes de chargement » des molécules $\mathrm{CMH}-$ I. La tapasine, une protéine transmembranaire ayant une structure similaire aux molécules $\mathrm{CMH}$, n'est pas seulement requise pour rapprocher les molécules de classe I dépourvues de peptides à la «source » de ces derniers, mais joue aussi un rôle important en optimisant les peptides présentés par les molécules de classe I. Dans les cellules déficientes en tapasine, le nombre et la stabilité des molécules $\mathrm{CMH}-\mathrm{I}$ à la surface sont réduits de façon importante. Le mécanisme de cette optimisation est encore mal compris, mais la rétention des molécules de classe I dépourvues de peptide par la tapasine, de concert avec la calréticuline, semble jouer un rôle important.

\section{Présentation croisée des antigènes exogènes par les molécules du CMH de classe I}

La présentation croisée (cross-presentation) désigne une voie de processing supplémentaire, propre

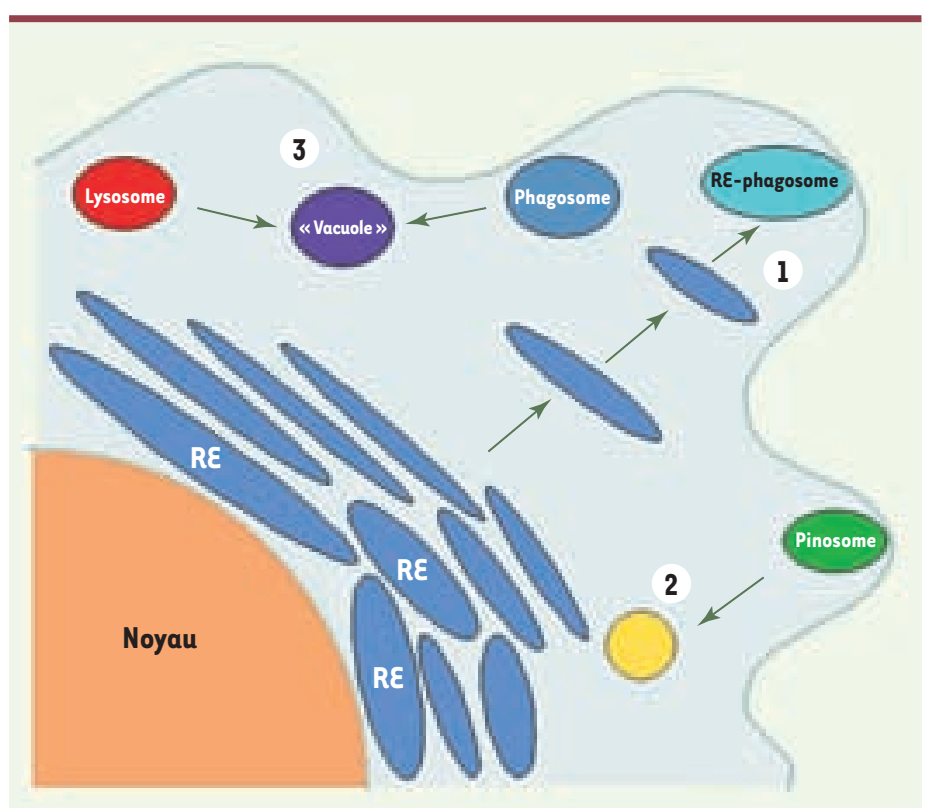

Figure 3. Voies hypothétiques de cross-presentation dans les cellules dendritiques. Les antigènes internalisés par phagocytose et pinocytose utilisent des voies différentes pour avoir accès à l'appareil de processing du réticulum endoplasmique. Selon le modèle du RE-phagosome (1), le recrutement de membranes du RE au phagosome précoce apporte les pompes TAP avec les complexes de chargement des molécules de classe I du CMH, et le protéasome; le RE-phagosome devient ainsi une vésicule autonome de processing. Les antigènes pinocytés accèdent au $R \varepsilon$ périnucléaire (2) soit de façon passive, avec le flux des membranes, soit par un transport actif. Les antigènes qui restent à l'intérieur du phagosome tardif peuvent être apprêtés via une troisième voie (3), qui fait appel aux hydrolases acides des lysosomes, et n'implique ni TAP, ni protéasome. aux cellules dendritiques, qui donne à des antigènes internalisés l'accès aux mécanismes décrits plus haut, permettant leur dégradation, transport et assemblage avec les molécules du CMH-I [4]. La cross-presentation permet aux cellules dendritiques de déclencher et de soutenir des réponses cellulaires cytotoxiques contre des agents pathogènes qui ne les infectent pas, et occupe donc une place importante dans l'immunité adaptative.

Par rapport au processing des protéines endogènes, cette voie se distingue donc par la communication entre la voie endosomique, responsable de l'acquisition des antigènes, et les compartiments impliqués dans leur processing, le cytosol et le RE. La nature précise de cette communication reste incertaine, mais plusieurs modèles ont été proposés [27] (Figure 3). Un premier modèle proposait l'existence d'un mécanisme de transport membranaire entre les endosomes et le cytosol, qui permettait aux antigènes internalisés d'accéder au pool d'antigènes cytosoliques. Un modèle plus récent, celui du «RE-phagosome», propose au contraire que le phagosome, formé avec un apport important de membranes et de protéines du $R \varepsilon$, fonctionnerait comme un compartiment spécialisé réunissant tous les éléments requis pour le processing des antigènes [28]. Cependant, une analyse rigoureuse n'a pas confirmé une contribution de membranes du RE à la formation du phagosome, mettant ainsi en doute le modèle du RE-phagosome [29]. Par ailleurs, ce modèle intègre difficilement le rôle important, dans la crosspresentation, du routage des molécules de classe I dans la voie endosomale [30]. En conclusion, il n'existe pas encore de modèle précis permettant de décrire les détails du mécanisme cellulaire de la cross-presentation.

\section{Conclusions}

La complexité du système de processing, doté d'éléments permettant le prélèvement d'échantillons sur la quasi-totalité des antigènes endogènes, mais également sur ceux extérieurs à la cellule présentatrice, répond à la complexité des périls auxquels la cellule fait face. La puissance de ce système est illustrée par la multitude des astuces employées par ses «adversaires» pour tenter d'échapper à sa surveillance. Ainsi, de nombreux virus, tout particulièrement ceux de la famille herpes, inhibent diverses étapes du processing, notamment celles du transport de peptides par les pompes TAP et de l'assemblage des complexes peptide/CMH-I dans le RE. Des phénomènes similaires $d^{\prime}$ ' échappement immunitaire » sont observés pour de nombreuses tumeurs, ce qui apporte des preuves supplémentaires de l'efficacité du système de processing. $\diamond$ 


\section{REMERCIEMENTS}

Tous mes remerciements à Lucienne Chatenoud pour ses suggestions quant à la rédaction de cet article.

\section{SUMMARY}

\section{Processing of MHC class I presented antigens}

The immune defences of our organism against pathogens and malignant transformation rely to a large extent on surveillance by cytotoxic $T$ lymphocytes. This surveillance in turn depends on the antigen processing system, which provides peptide samples of the cellular protein composition to MHC (major histocompatibility complex) class I molecules displayed on the cell surface. To continuously and almost in real time provide a representative sample of the array of proteins synthesized by the cell, this system exploits some fundamental pathways of the cellular metabolism, with the help of several dedicated players acting exclusively in antigen processing. Thus, a key element in the turnover of cellular proteins, protein degradation by cytosolic proteasome complexes, is exploited as source of peptides, by recruiting a minor fraction of the produced peptides as ligands for MHC class I molecules. These peptides can be further processed and adapted to the precise binding requirements of allelic MHC class I molecules by enzymes in the cytosol and endoplasmic reticulum. The latter compartment is equipped with several dedicated players helping peptide assembly with class I molecules. These include the TAP (transporter associated with antigen processing) membrane transporter pumping peptides into the $\varepsilon R$, and tapasin, a chaperone with a structure similar to MHC molecules that tethers class I molecules awaiting peptide loading to the TAP transporter, and mediates optimization of MHC class I ligand by a still somewhat mysterious mechanism. Additional "house-keeping" chaperones that are known to act in concert in $\varepsilon R$ quality control, assist and control correct folding, oxidation and assembly of MHC class I molecules. While this processing system handles exclusively endogenous cellular proteins in most cells, dendritic cells employ one or several special pathways to shuttle exogenous, internalized proteins into the system, in a process referred to as cross-presentation. Deciphering the cell biological mechanism creating the link between the endosomal and secretory pathways that enables cross-presentation is one of the challenges faced by contemporary research in the field of MHC class I antigen processing. $\diamond$

\section{RÉFÉRENCES}

1. Janeway C. Immunobiology: the immune system in health and disease, $6^{\text {th }}$ ed. New York: Garland Science, 2005.

2. Shastri N, Schwab S, Serwold T. Producing nature's gene-chips : the generation of peptides for display by MHC class I molecules. Annu Rev Immunol $2002 ; 20: 463-93$.

3. Saveanu L, Carroll 0, Hassainya Y, van Endert P. Complexity, contradictions, and conundrums : studying post-proteasomal proteolysis in HLA class I antigen presentation. Immunol Rev $2005 ; 207: 42-59$.

4. Wilson NS, Villadangos JA. Regulation of antigen presentation and cross-presentation in the dendritic cell network : facts, hypothesis, and immunological implications. Adv Immunol $2005 ; 86: 241-305$.

5. Ciechanover A. Proteolysis : from the lysosome to ubiquitin and the proteasome. Nat Rev Mol Cell Biol 2005; $6: 79-87$

6. Baumeister $W$, Walz J, Zuhl F, Seemuller $\varepsilon$. The proteasome : paradigm of a selfcompartmentalizing protease. Cell 1998; $92: 367-80$.
7. Meusser B, Hirsch C, Jarosch $\varepsilon$, Sommer T. ERAD : the long road to destruction. Nat Cell Biol $2005 ; 7$ : 766-72.

8. Yewdell J. To DRiP or not to DRiP : generating peptide ligands for MHC class I molecules from biosynthesized proteins. Mol Immunol 2002 ; 39: 139-46.

9. Vabulas RM, Hartl FU. Protein synthesis upon acute nutrient restriction relies on proteasome function. Science $2005 ; 310$ : 1960-3.

10. Fruci D, Lauvau G, Saveanu L, et al. Quantifying recruitment of cytosolic peptides for HLA class I presentation : impact of TAP transport. J Immunol 2003; 170 : 2977-84.

11. Princiotta MF, Finzi D, Qian SB, et al. Quantitating protein synthesis, degradation, and endogenous antigen processing. Immunity 2003 ; $18: 343-54$.

12. Schwab SR, Shugart JA, Horng T, et al. Unanticipated antigens: translation initiation at CUG with leucine. PLoS Biol $2004 ; 2$ : e366.

13. Vigneron N, Stroobant V, Chapiro J, et al. An antigenic peptide produced by peptide splicing in the proteasome. Science $2004 ; 304: 587-90$.

14. Ciechanover $A$. The ubiquitin-proteasome pathway: on protein death and cell life. EMBO J $1998 ; 17: 7151-60$.

15. Kloetzel PM. Generation of major histocompatibility complex class I antigens : functional interplay between proteasomes and TPPII. Nat Immunol 2004 ; 5 : 661-9.

16. Kisselev AF, Akopian TN, Woo KM, Goldberg AL. The sizes of peptides generated from protein by mammalian 26 and $20 \mathrm{~S}$ proteasomes. Implications for understanding the degradative mechanism and antigen presentation. J Biol Chem 1999; 274 : 3363-71.

17. Reits $\varepsilon$, Neijssen J, Herberts $C$, et al. A major role for TPPII in trimming proteasomal degradation products for $\mathrm{MHC}$ class I antigen presentation. Immunity $2004 ; 20: 495-506$.

18. Saric T, Graef Cl, Goldberg AL. Pathway for degradation of peptides generated by proteasomes: a key role for thimet oligopeptidase and other metallopeptidases. J Biol Chem 2004 ; 279 : 46723-32.

19. Srivastava PK, Udono H, Blachere NE, Li Z. Heat shock proteins transfer peptides during antigen processing and CTL priming. Immunogenetics $1994 ; 39: 93-8$.

20. van Endert PM, Saveanu L, Hewitt EW, Lehner P. Powering the peptide pump : TAP crosstalk with energetic nucleotides. Trends Biochem Sci $2002 ; 27: 454-61$.

21. Momburg F, Neefjes JJ, Hämmerling G. Peptide selection by MHC-encoded TAP transporters. Curr Opin Immunol $1994 ; 6: 32-7$.

22. Van Endert PM, Riganelli D, Greco G, et al. The peptide-binding motif for the human transporter associated with antigen processing. J Exp Med $1995 ; 182$ : 1883-95.

23. Rock KL, York IA, Goldberg AL. Post-proteasomal antigen processing for major histocompatibility complex class I presentation. Nat Immunol $2004 ; 5: 670-7$.

24. Saveanu L, Carroll 0, Lindo V, et al. Concerted peptide trimming by human ERAP1 and ERAP2 aminopeptidase complexes in the endoplasmic reticulum. Nat Immunol $2005 ; 6: 689-97$.

25. Chang SC, Momburg F, Bhutani N, Goldberg AL. The $\varepsilon R$ aminopeptidase, ERAPl, trims precursors to lengths of MHC class I peptides by a «molecular ruler » mechanism. Proc Natl Acad Sci USA 2005; 102 : 17107-12.

26. Cresswell P, Bangia N, Dick T, Diedrich G. The nature of the MHC class I peptide loading complex. Immunol Rev 1999; 172 : 21-8.

27. Saveanu L, van Endert P. Dendritic cells : open for presentation business. Nat Immunol $2005 ; 6: 7-8$.

28. Desjardins M. $\varepsilon$ R-mediated phagocytosis : a new membrane for new functions. Nat Rev Immunol 2003; $3: 280-91$.

29. Touret $N$, Paroutis $P$, Terebiznik M, et al. Quantitative and dynamic assessment of the contribution of the $\varepsilon R$ to phagosome formation. Cell $2005 ; 123: 157-70$.

30. Lizee G, Basha G, Tiong J, et al. Control of dendritic cell crosspresentation by the major histocompatibility complex class I cytoplasmic domain. Nat Immunol 2003 ; 4 : 1065-73.

\section{TIRÉS À PART}

P. van Endert 